Y. Shikata and W. Klingenberg

Nagoya Math. J.

Vol. 100 (1985), 65-81

\title{
ON A PROOF OF DIVISIBILITY LEMMA, I
}

\author{
Y. SHIKATA AND W. KLINGENBERG
}

\section{Introduction}

A method to show the existence of infinitely many closed geodesics on a manifold with non degenerate Riemannian metric is to use socalled divisibility lemma which is conjectured to hold in [K].

Our purpose in this series of papers is, then, to show first the divisibility lemma in a modified form on $k$-sphere $S^{k}(k \geqq 3)$ with (strongly) non degenerate Riemannian metric and to deduce the existence of infinitely many closed geodesics on $S^{k}(k \geqq 3)$ using the modified divisibility lemma by equivariant modifications of flows.

In the present paper, we prepare several algebraic tools to prove the modified divisibility lemma and in the next paper(s) we give necessary geometric construction to apply the algebraic tools on it and complete the proof of the divisibility lemma in a modified form.

The algebraic tools introduced in this note are Morse complex, barycenter $B_{c}$ and cycle $Z(c)$ over a critical point $c$.

Though we start this note with abstract Morse complex, it is in reality defined to be a chain complex over critical manifold of 0 or 1 dimension for a manifold with $S^{1}$ action and an invariant strongly non degenerate energy function.

In the chain group of Morse complex, there is defined a natural splitting $S_{n} \oplus T_{n}$ by the subgroup $S_{n}$ generated over critical points and the subgroup $T_{n}$ generated over critical submanifolds of 1 dimension.

The divisibility property, that is, the possibility to find a critical point $c^{\prime}$ for a given critical point $c$ such that $m\left(c^{\prime}\right)$, the order of $c^{\prime}$, divides that of $c$;

$$
m\left(c^{\prime}\right) \mid m(c)
$$

then, is translated into a cycle and boundary problem by the notion 0 :

Received April 3, 1984.

Revised January 29, 1985. 
barycenter $B_{c}$ over $c$ and the cycle $Z(c)$.

The barycenter $B_{c}(x)$ is a homomorphism of the chain group into the (additive) complex number space commuting with the natural $S^{1}$ actions.

The essential property of the barycenter is that we have

$$
m\left(c^{\prime}\right) \mid m(c)
$$

if $B_{c}\left(\partial c^{\prime}\right) \neq 0$ for critical points $c, c^{\prime}$.

This may be generalized by considering a cycle $Z$ in Morse complex with non zero barycenter over $c$ and a bounding chain $Y=Y(S)+Y(T)$ $(Y(S) \in S, Y(T) \in T)$ such that $B_{c}(\partial Y(T))=0$ as in Theorem 1.1, which essentially asserts the algebraic divisibility.

In the final section of this note, we construct a cycle $Z(c) \in S_{n}$ over a critical point $c$, in case of the closed path space over $S^{k}$, if $Z(c)$ is non zero in $H\left(\Lambda\left(S^{n}\right)\right)$, we find a zero homologous cycle $Z$ easily with non zero barycenter over $c$, making use of the orientation reversing map $\vartheta$.

Thus we see that the divisibiliy lemma may be obtained under an assumption that we can modify the bounding chain $Y$ of $Z$ so as to satisfy its $T$-component $Y(T)$ is such that $B_{c}(\partial(Y(T)))=0$.

The modification is done by a geometric method under the assumption that the fundamental group of the closed path space is zero and will be done in the next paper (see also Appendix).

We start Section 1 of the present note with abstract Morse complex and supply in Section 2 its geometric aspect on the space of closed curves $\Lambda(M)$ on a Riemannian manifold $M$ provided that $\Lambda(M)$ has an $S^{1}$ invariant (strongly) non degenerate energy function.

There we find an $S^{1}$ equivariant map $K$ of a class of submanifolds with boundary into the Morse complex.

Though we do not go into full detail, an expository work on them may be found in $[\mathrm{H}]$. Since $K$ commutes with the boundary operators, we may consider the map $K$ as a homomorphism of $L$-equivalence class of submanifolds into the homology of the Morse complex (for detail see Fukazawa's master thesis).

The homomorphism $K$, which is called $K$-decomposition, has its inverse operator $H$ obtained from handle body construction.

Though our original plan was as above, we decided to include in this paper as appendix a rough sketch of the modification above, socalled tunnel killing process, to meet several requests we received. 
Also the remarks in the present paper contain the topics which will be treated in the coming paper.

We thank deeply to Prof. K. Grove, N. Kuiper, M. Nakaoka and D. Sullivan for valuable discussions and for warm encouragement also to Dr. T. Ozawa.

\section{§1. Abstract Morse complex}

We consider $S^{1}$ as the quotient group of the real number $R$ by the integer $Z$ and we denote by $\alpha \circ x$ the action of $\alpha \in S^{1}$ on $x \in S$ for a set $S$ with $S^{1}$ action.

Take the projection image $\{I\}$ of all the intervals $\{[\alpha, \beta), \alpha \neq \beta\}$ in $R$ by the covering map $p$ of $R$ onto $S^{1}$. Then the set of the formal elements $I \circ s(I \in\{I\}, s \in S)$ turns out to be a set with $S^{1}$ action by defining

$$
\gamma \circ(I \circ s)=I \circ(\gamma \circ s), \quad \gamma \in S^{1} .
$$

Among the set $\{I \circ s\}$ of the formal elements, we introduce an equivalence relation $\sim$ using the covering coordinate;

$$
\gamma \circ(p[\alpha, \beta)) \circ s) \sim p([\alpha+\tilde{\gamma}, \beta+\tilde{\gamma})) \circ s
$$

for $p(\tilde{\gamma})=\gamma$.

If there occurs no confusion, we identify $\tilde{\gamma}$ and $\gamma$ in what follows.

The set $\{I \circ s\} / \sim=T=T(S)$ is well defined and have an $S^{1}$ action induced from that on $\{I \circ s\}$.

In case that we are given a sequence $\left\{S_{n}\right\}$ of sets with $S^{1}$ action, we set

$$
T_{n}=T\left(S_{n-1}\right) \text {. }
$$

The free abelian groups over $S_{n}$ and $T_{n}$ have natural $S^{1}$ action and will be denoted again by $S_{n}$ and $T_{n}$, respectively.

For the sake of simplicity, we may divide $T_{n}$ by the usual degeneracy to have the following relation, we still use $T_{n}$ for the quotient:

$$
\begin{aligned}
& p([\alpha, \beta)) \circ s+p([\beta, \gamma)) \circ s=p([\alpha, \gamma)) \circ s \\
& p([\alpha, \beta)) \circ\left(s_{1}+s_{2}\right)=p([\alpha, \beta)) \circ s_{1}+p([\alpha, \beta)) \circ s_{2} \\
& p([\alpha, \beta)) \circ\left(p\left(\left[\alpha^{\prime}, \beta^{\prime}\right)\right) \circ s\right)=0
\end{aligned}
$$

Assume that we are given a boundary $\partial$ fo $S_{n}$ into $S_{n-1} \oplus T_{n-1}$ commuting with $S^{1}$ action and extend it to $T_{n+1}$ by 


$$
\partial(p([\alpha, \beta)) \circ s)=p(\alpha) \circ s-p(\beta) \circ s-p([\alpha, \beta)) \circ \partial s .
$$

Then $\left(S_{n} \oplus T_{n}, \partial\right)$ turns out to be a chain complex with $S^{1}$ action.

The chain complex obtained in this way from sets $\left\{S_{n}\right\}$ and boundary $\partial$ is defined to be an abstract Morse complex (with $S^{1}$ action) associated to $\left\{S_{n}\right\}$ and $\partial$. The element in $S_{n}$ or in $T_{n}$ is said to be summit or tunnel, respectively.

Denote by $I(x)$ the isotropy group of $x \in S_{n} \oplus T_{n}$;

$$
I(x)=\left\{g \in S^{1} / g \circ x=x\right\} .
$$

The order ord $(I(x))$ of $I(x)$ is called the (abstract) multiplicity $m(x)$ of $x$. We assume here $m(c)$ is finite for any base $c \in S_{n}$.

Lemma 1. From the definition, it is obvious that $m(c)=m([\alpha, \beta) \circ c)$ for $c \in S$ except for the case $\alpha \circ c=\beta \circ c$.

For a base $c$ in $S_{n}$, the small circle $\sigma c$ on $c$ is a subset of basis of $S_{n}$ defined by

$$
\sigma(c)=\{g \circ c / 0 \leqq g<1 / m(c)\}
$$

For an arithmetic sequence

$$
\left\{\alpha_{i}=i / k m(c)+\beta, i=1, \cdots, k\right\}
$$

the set of summit basis $\left\{\alpha_{i} \circ c / i=1 \cdots k\right\}$ or tunnel basis $\left\{\alpha_{i} \circ I \circ c / i=1 \cdots k\right\}$ $(I \in\{I\})$ is said to be evenly distributed on $\sigma(c)$.

Obviously for $e=\beta \circ c$ or $I \circ c$ the evenly distributed $k$ elements $\left\{\alpha_{i} \circ e / i=1 \cdots k\right\}$, is invariant under the action of $p / k m(s) \in S^{1}$ for any natural number $p$. Thus the sum $\sum_{1}^{k} \alpha_{i} \circ e \in S_{n} \oplus T_{n}$ is also invariant under the action of $p / k m(c)$ and is called the elementary invariant of order $k$ over $c$.

Lemma 2. If $x \in S_{n} \oplus T_{n}$ is invariant under a finite group $G \subset S^{1}$, then $x$ decomposes into a sum of elementary invariants each of which is invariant under $G$.

In fact, we see first the component $x_{c}$ of $x$ over the basis system on the small circle $\sigma c$ of $c$ is $G$ invariant for any $c$. We see also the set of basis which appears in $x_{c}$ is $G$ invariant and therefore splits into a disjoint union of $G$ invariant sets $A^{j}=\left\{\alpha_{i}^{j} \circ e\right\}$ on which $G$ acts transitively.

Thus the invariance of $x_{c}$ implies the component of $x_{c}$ over the basis $A^{j}$ has the same coefficient, indicating that 


$$
x=\sum_{o c}\left(\sum_{j} a_{j}\left(\sum_{l} \alpha_{1}^{j} \circ e\right)\right) \quad a_{j} \in Z,
$$

where we use $\sum_{\sigma c}$ to symbolize the sum over summit $e=\beta \circ c$ or tunnel $e=I \circ c$ lying on $\sigma c$,

Corollary 1. For any $x \in S_{n} \oplus T_{n}$, $\partial x$ also decomposes into a sum of elementary invariants each of which is invariant under the isotropy $I(x)$ of $x$.

For a base $c \in S_{n}$ define a map $B_{c}$ of the small circle $\sigma c$ over $c$ onto the unit circle of center zero in the complex number space by

$$
B_{c}(\alpha \circ c)=\exp (2 \pi \sqrt{-1} m(c) \alpha) .
$$

Extend the map $B_{c}$ to a linear map over $Z$ of $S_{n} \oplus T_{n}$ into the complex number space, setting $B_{c}(e)=0$ for any base $e \notin \sigma c$ or $e \in T_{n}$.

The map $B_{c}$ is obviously well defined and has the following property:

Lemma 3. $B_{c}$ is linear over $Z$ and satisfies that

$$
B_{c}(\alpha \circ x)=\exp (2 \pi \sqrt{-1} m(c) \alpha) B_{c}(x) .
$$

The image $B_{c}(x)$ of $x \in S_{n} \oplus T_{n}$ is called the barycenter of $x$ (over $c$ ).

Corollary 2. For the elementary invariant $y_{k}$ of order $k y_{k}=$ $\sum_{i} i / k m(c) \circ c$, the barycenter $B_{c}\left(y_{k}\right)$ is 1 if $k=1$ and is 0 if $k>1$.

The case $k=1$ is obvious and for $k>1$ we see that the set consisting of the elements

$$
B_{c}(i / k m(c) \circ c)=\exp (2 \pi \sqrt{-1} i / k) \quad i=1, \cdots, k,
$$

is nothing but the set of $k$-th roots of unity. Therefore the coefficient of $(k-1)$-th term of the equation $x^{k}-1=0$ agrees with the sum

$$
-B_{c}\left(\sum i / k m(c) \circ c\right)=-\sum B_{c}(i / k m(c) \circ c) \text {. }
$$

Since any elementary invariant element $x$ of order $k$ over $c$ is obtained as the shift of $y_{k}$, that is, $x=\alpha \circ y_{k}$ for some $k$ and $\alpha \in S^{1}$, we have

Corollary 3. For any elementary invariant $x$ over $c$, it holds that $B_{c}(x) \neq 0$, if and only if elementary invariant $x$ is of order 1 , in other words,

$$
x=\alpha \circ c, \quad \alpha \in S^{1}
$$


It follows easily from Corollaries 1 and 3 that $B_{c}(\partial x) \neq 0$ implies $c$ is invariant under $I(x)$, that is, $I(c)$ contains $I(x)$ as a subgroup, yielding that $m(x)$ divides $m(c)$. Here we have to notice that like in case $x$ itself is a tunnel piece $I \circ c$ over $c$ for example, the statement above might happen to say nothing special.

Therefore we rewrite it as follows:

Proposition 1. For a summit element $x \in S_{n+1}$ suppose there exists a summit base $c \in S_{n}$ such that $B_{c}(\partial x) \neq 0$, then there is found a summit base $c^{\prime} \in S_{n+1}$ such that $c$ belongs to $\partial c^{\prime}$ and $m\left(c^{\prime}\right)$ divides $m(c)$.

Take a chain $x \in S_{n} \oplus T_{n}$ and an elementary decomposition of $\partial x$ of Lemma 2, which we write

$$
\partial x=\sum_{\sigma c}\left(\sum a_{j}\left(\sum_{i} \alpha_{i}^{j} \circ e\right)\right),
$$

using the notation in Lemma 1.

Take then the set $\{k m(c)\}$ of the order times the multiplicity of elementary invariants in the decomposition above and introduce a partial order $\prec$ in the set by

$$
p_{1}>p_{2} \quad \text { if and only if } p_{2} \mid p_{1} .
$$

We notice here that for an elementary invariant $\sum \alpha_{i} \circ e$ of order $k$ over $c$, it holds that

$$
\sum \alpha_{i} \circ e=1 / p \circ\left(\sum \alpha_{i} \circ e\right)
$$

for any $p \prec k m(e)$. Thus the set $P=\left\{p_{1}, \cdots, p_{e}\right\}$ of minima in the set $\{k m(c)\}$ relative to the order $\prec$ satisfies that

$$
\begin{aligned}
0 & =\left(\prod_{P}\left(1 \ominus 1 / p_{i}\right)\right) \circ \partial x \\
& =\left(1 \ominus 1 / p_{1}\right) \circ\left(1 \ominus 1 / p_{2}\right) \circ \cdots \circ\left(1 \ominus 1 / p_{e}\right) \circ \partial x
\end{aligned}
$$

where $(1 \ominus 1 / p) \circ y$ stands for $y-1 / p \circ y$.

Therefore we have

Lemma 4. For an $x \in S_{n} \oplus T_{n}$, there corresponds a cycle $Z(x)$ which is of the form

$$
Z(x)=\prod\left(1 \ominus 1 / k_{i} m\left(e_{i}\right)\right) \circ x,
$$

where $k_{i}, m\left(e_{i}\right)$ are the order and the multiplicity of elementary invariants 
in $\partial x$, respectively.

Direct computation using Lemma 3 yields

LEMMA 5. If $c$ is a summit base, the barycenter over $c$ of the cycle $Z(c)$ is as follows:

$$
B_{c}(Z(c))=\prod\left(1-\exp \left(2 \pi \sqrt{-1} m(c) / k_{i} m\left(c_{i}\right)\right)\right)
$$

Corollary 4. For a summit base $c, B_{c}(Z(c))$ is not zero if in the decomposition of $\partial c$ there is no elementary invariant e whose multiplicity $m(e)$ divides $m(c)$.

We say a cycle $x \in S_{n} \oplus T_{n}$ is connected if there are no non zero cycles $x_{1}, x_{2}$ so that $x=x_{1}+x_{2}$.

Corollary 5. For any summit base c, the cycle $Z(c)$ above is connected.

Now we can formulate the first step of the divisibility as follows:

Theorem 1. For any summit base $c \in S_{n}$ we have the following two cases:

1) There appears a summit base $c_{-} \in S_{n-1}$ or a tunnel base $I \circ c_{--}\left(c_{--} \in S_{n-2}\right)$ on the boundary $\partial c$ such that

$$
m\left(c_{-}\right) \mid m(c) \quad \text { or } \quad m\left(c_{--}\right) \mid m(c) \text {. }
$$

2) Otherwise we have non zero cycle $Z(c)$ of non zero barycenter over $c$. Therefore if $Z(c)$ or $Z(c)+y$ is bounded by a summit element $x$ for some $y$ with barycenter zero over $c$, then there is found a summit base $c^{\prime} \in S_{n+1}$ such that

$$
m\left(c^{\prime}\right) \mid m(c)
$$

\section{§2. Morse theory on the path space}

We review quickly here some of the notions and notations from the Morse theory $(\Lambda=\Lambda(M), E)$ on the space $\Lambda$ of non trivial $H^{1}$ closed curves on a Riemannian manifold $M$ and the energy function $E$ on $A$. ([K]).

If Riemannian metric is non degenerate, then the (non-trivial) critical point of $E$ agrees with the closed geodesic in $M$ and for each critical point $c$ there are associated the strongly stable, manifold $S(c)$ and the strongly unstable manifold $U(c)$ which are open submanifolds in $\Lambda$ : 


$$
\begin{aligned}
& S(c)=\left\{x / \lim _{t \rightarrow+\infty} \varphi_{t}(x)=c\right\} \\
& U(c)=\left\{x / \lim _{t \rightarrow-\infty} \varphi_{t}(x)=c\right\}
\end{aligned}
$$

where $\varphi_{t}$ is the flow on $\Lambda$ obtained from $-\operatorname{grad} E$.

Being $\Lambda$ interpreted as a subspace of the mapping space of $S^{1}$ into $M$, the group $S^{1}$ and $Z_{2}$ act on $\Lambda$ through the parameter shift or inversion:

$$
\begin{aligned}
& \theta \circ \alpha(t)=\alpha(\tilde{t}+\tilde{\theta}) \\
& \vartheta \circ \alpha(t)=\alpha(-\tilde{t}), \quad\left(\alpha \in \Lambda, t, \theta \in S^{1}\right)
\end{aligned}
$$

The set $S^{1} \circ S(c)=\cup_{\theta \in S^{1}} \theta \circ S(c)$ covers the stable manifold $\sigma(S(c))$ and it holds that

$$
\begin{aligned}
\operatorname{dim} U(c) & =\operatorname{index} c=\operatorname{codim} \sigma(S(c)) \\
\operatorname{codim} S(c) & =\operatorname{index} c+1 .
\end{aligned}
$$

We take the set of the critical points of index $n$ together with an orientation in $U(c)$ to be the set $S_{n}$ in Section 1 , then we contract the abstract Morse complex over the set of the critical points.

Thus we identify the summit base $c$ to a geometric critical point in $A$ or more geometrically, to the (oriented) unstable manifold $U(c)$ around $c$ and we can speak of (geometric) tunnel piece and the small circle. The abstract tunnel piece $[\alpha, \beta) \circ c$ over $c$ may be considered as a set of critical points

$$
[\alpha, \beta) \circ c=\bigcup_{\alpha \leq t<\beta} t \circ c
$$

or as a submanifold (covered by)

$$
[\alpha, \beta) \circ U(c)=\bigcup_{\alpha \leq t<\beta} t \circ U(c),
$$

and the small circle $\sigma c$ may be considered as a circle given by

$$
\sigma c=\bigcup_{0 \leq t<1 / m(c)} t \circ c
$$

The gradient flow defines a map $\pi$ of $\sigma(S(c))$ onto the small circle $\sigma c$. The map $\pi$ defines a correspondence of the set of points in $\sigma(S(c))$ into the set of summit basis on $\sigma c$ and also that of the set of curves in $\sigma(S(c))$ into the set of tunnel basis on $\sigma c$. We notice that

LEMmA 1. If an n-submanifold $X$ of $\Lambda$ (may have boundary and be 
non connected) is transversal to every stable submanifold $\sigma(S(c))$, then $X$ splits into a disjoint union of intersections $X \cap \sigma(S(c))$;

$$
X=\bigcup_{\sigma c}(X \cap \sigma(S(c))),
$$

and the tangent space $T_{P}(X)$ of $X$ at $P \in X \cap \sigma(S(c))$ splits into two parts:

$$
T_{P}(X)=U \oplus V
$$

where $U$ projects onto the tangent space of $U(\pi(X \cap \sigma(S(c)))$ and $V$ projects down to zero by $\pi_{*}$.

In particular for a transversal submanifold $X$ and for critical point $c, c_{-}$of index $n, n-1$, respectively, we have that

$$
\begin{aligned}
& X \cap \sigma(S(c))=\{\text { points }\} \\
& X \cap \sigma\left(S\left(c_{-}\right)\right)=\{\text {curves }\} .
\end{aligned}
$$

Hence by $\pi$ we have a correspondence $\tilde{K}_{S}, \tilde{K}_{T}$ of transversal $n$-submanifold into the set of basis in $S_{n}, T_{n}$ respectively, discarding another intersection then these giving $n$-dimensional basis. The correspondence $\tilde{K}_{S}, \tilde{K}_{T}$ yields naturally a correspondence $K_{S}, K_{T}$ into the $n$-chain group $S_{n}, T_{n}$, respectively. Finally, $K=K_{S} \oplus K_{T}$ gives a correspondence into the group $S_{n} \oplus T_{n}$.

Lemma 2. All the correspondences $\tilde{K}_{s}, K_{S}, \tilde{K}_{T}, K_{T}, K$ commute with $S^{1}$ action and relative to the disjoint union of submanifolds $K_{S}, K_{T}, K$ turn out to be homomorphism.

Definition. Riemannian metric on $M$ (and the induced energy function $E$ on $\Lambda(M)$ is said to be strongly non degenerate if it is non degenerate and if the strongly unstable manifold $U(c)$ is transversal to any stable manifold $\sigma\left(S\left(c^{\prime}\right)\right)$ for every critical points $c, c^{\prime}$.

Remark. For our purpose, it may not be strictly necessary to have strongly non degenerate Riemannian metric, because we may twist the energy function induced from non degenerate Riemannian metric so as to be $S^{1}$ invariant, strongly non degenerate and to have the same critical point as the original energy function.

Though we shall return in the coming paper to this problem of removal of the strong non degeneracy assumption, we sketch here roughly how it should be done: Suppose that the unstable manifold $U(c)$ of $c$ is 
not transversal to a stable manifold $\sigma\left(S\left(c^{\prime}\right)\right)$ of $c^{\prime}$ with $E\left(c^{\prime}\right)<E(c)$. We may consider these manifolds are in the tangent space of $c$, where $U(c)$ appears as a linear space orthogonal to the $S^{1}$ action. Then the non transversal intersection is on the boundary $\partial D_{\varepsilon}$ of $\varepsilon$ disk $D_{\varepsilon}$ around 0 in the tangent space and is orthogonal to the $S^{1}$ action. Therefore we may twist the tangent space keeping the direction of the $S^{1}$ action fixed so as to the intersection is transversal including the finite action of isotropy group.

Since the twisting can be chosen to be diffeotopic to the identity by a suitable diffeotopy $h_{t}$, we define energy function $E$ as follows:

$$
\tilde{E}= \begin{cases}h_{\varepsilon(1-t)}^{*} E & \text { on } D_{c t}^{\perp} \\ E \text { outside of } D_{\dot{t}}^{\perp}\end{cases}
$$

where $D_{\varepsilon}^{\perp}$ is the $\varepsilon$ disk of codimension 1 in $D_{\varepsilon}$ orthogonal to the $S^{1}$ action. And we extend $\tilde{E}$ trivially onto $\sigma D_{\varepsilon}$ by the $S^{1}$ action, which is possible by the invariance of $E$ under the $S^{1}$ action.

Therefore without loss of generality, we may assume the strong non degeneracy in what follows, under the non degeneracy assumption.

We define boundary $\partial c$ of a critical point $c \in S_{n}$ as follows:

Take a small $n$-disk $D(c)$ around $c$ in the strongly unstable manifold $U(c)$ so that its (geometric) boundary $\partial D(c)$ is transversal to the gradient flow and set

$$
\partial c=K(\partial D(c))
$$

Lemma 3. The definition above is independent of the choice of $D$ and therefore $\partial$ is well-defined.

In fact, the transversality with the gradient flow implies that the flow gives a diffeomorphism between $D$ and a standard transversal sphere $N$ around $c$ obtained from $\varepsilon$-sphere in the negative boundle.

Since $U(c) \cap \sigma\left(S\left(c_{-}\right)\right)$contains the flow from $c$ to $\sigma \circ c_{-}$as long as it is not empty, the diffeomorphism gives the 1-1 correspondence between the intersection of $\sigma\left(S\left(c^{\prime}\right)\right)$ with $\partial D$ and with $N_{\varepsilon}$.

Corollary 1. o commutes with the $S^{1}$ action.

Let $X$ be a compact closed submanifold in $A M$ transversal to every stable, unstable manifolds. We assume further that for each $p \in X \cap \sigma(S(c))$ there exists a neighbourhood $N(p)$ in $X$ which covers $D(c)$ under the flow, 
which we refer $X$ to be in the nice position.

It is obvious that if $\partial D(c)$ intersects with $\sigma S\left(c_{-}\right)$(ind $c_{-}=n-1$ ), then $\partial N(p)$ also intersects with it and therefore $X$ has 1 dimensional intersection with $\sigma S\left(c_{-}\right)$.

Take any non closed connected component $\gamma$ of the 1 dimensional intersection $X \cap \sigma S\left(c_{-}\right)$which does not contain any intersection with $\sigma S(\tilde{c})$ (ind $\tilde{c}=n$ ) except both ends. Then we see that $\gamma$ ends with critical points $c_{1}, c_{2}$ of index $n$ and that $\gamma$ has point intersections with $N\left(c_{1}\right), N\left(c_{2}\right)$, which make a part of the boundary component of $\partial c_{1}, \partial c_{2}$. Obviously $\gamma$ corresponds to a tunnel piece over $c_{-}$and the summit part of $\partial r$ cancels the boundary component above from $\partial c_{1}, \partial c_{2}$.

Thus in this manner we see that the summit components of $\{\partial c\}$ of critical points $\{c\}$ of index $n$ in $X$ should be cancelled directly or by tunnel pieces in $X$ in general. In a similar way, we see that the tunnel pieces of $\{\partial c\}$ are cancelled and therefore we see that $\partial K(X)=0$ for a closed submanifold $X$ sitting in a nice position. Since we can show that any transversal $X$ may be twisted into the nice position keeping $K(X)$ fixed, we may concluded that $\partial \partial c=0$ taking $X$ to be (the set theoretical) boundary $\partial D(c)$ of $D(c)$.

Lemma 4. On $S_{n}, \partial$ is a differential operator, that is, $\partial \circ \partial=0$ on $S_{n}$

Proposition 1. We can associate cannonically an abstract Morse complex to the Morse theory on $(\Lambda, E)$ provided the non degenerate condition for $(\Lambda, E)$. The summit base of the complex corresponds to the critical point such that the dimension and the multiplicity in the Morse complex agrees with the index in the ordinary sense and the multiplicity except factor 2, respectively.

Only the multiplicity part may need explanation. Since the orientation in $U\left(c^{\prime}\right)$ is taken into consideration, the order of isotropy of a critical point $c$ may differ by factor 2, when $c$ is consider only as a point and when $c$ is considered together with orientation in the unstable manifold $U(c)$, resulting the difference of factor 2 at most in the multiplicities.

If we take a submanifold $X$ with boundary $\partial X$ in the argument above instead of a closed submanifold, we see that the curve $\gamma$ obtained as

$$
\gamma=X \cap \partial\left(S\left(c_{-}\right)\right), \quad \text { ind }\left(c_{-}\right)=\operatorname{dim} X-1
$$

may end in $\partial X$ and that the totality of these points agrees with the 
summit part of $\partial(K(X))$. Since a similar argument yields this holds also for the tunnel part, we have

Proposition 2. For a transversal submanifold $X$ in $A$ it holds that

$$
\partial K(X)=K(\partial X) \text {. }
$$

Thus operator $K$ gives a natural correspondence of the set of transversal submanifolds into the chain group of the Morse complex (see also Lemma 2).

We also can construct a natural correspondnece $J$ of the chain group into the set of submanifolds as follows: First for a summit base $c \in S_{n}$, let $\bar{J}(c)=D(c)$ where $D(c)$ is the (oriented) $n$-disk in $U(c)$ centered at $c$ and next for a tunnel $[\alpha, \beta) \circ c$, let $\bar{J}([\alpha, \beta) \circ c)=\bigcup_{\alpha \leq t<\beta} t \circ D(c)$, then for a chain $x=\sum a_{i} e_{i}$ define $\bar{J}(x)$ to be the disjoint union of $a_{i}$ copies of $\bar{J}\left(e_{i}\right)$. Finally $J(x)$ is given as a submanifold (may not be connected) without intersection obtained from $\bar{J}(x)$ in a canonical way, so as to keep $K$ image fixed, making use of the dimensionality of $\Lambda$.

It is obvious that

Lemma 5. $K J(x)=x$ for any $x \in C_{n}$.

\section{§3. Relations to homology}

We review quickly a proof that the homology of the abstract Morse complex associated to the Morse theory $(\Lambda, E)$ is isomorphic to that of the path space $\Lambda$ under the non degenerate condition referred in page 73 .

The non degeneracy admits us to decompose $\Lambda$ into unstable manifolds $\sigma U(c)$ and therefore into $X_{i+1}$ given by

$$
\begin{aligned}
\sigma U(c) & =\bigcup_{0 \leq t<1 / m(c)} t \circ U(c), \\
X_{n+1} & =\bigcup_{\text {ind } c=n} \sigma U(c)
\end{aligned}
$$

so that

$$
\partial(\sigma U(c)) \subset \bigcup_{i \leq n} X_{i}, \quad \text { ind } c=n
$$

(see Lemma 1, § 2)

Thus we see that the boundary definition in Section 2 agrees with the boundary as the cell complex for each cell $\sigma U(c)$.

TheOREM 1. Under the non degeneracy assumption which says the 
transversality of stable and unstable manifolds, we have a canonical isomorphism of homology groups between $\Lambda$ and the abstract Morse complex associated to the Morse theory $(\Lambda, E)$.

The integral homology of the space $\Lambda=\Lambda\left(S^{n}\right)$ for $n$ sphere $S^{n}$ is computed by Schwarz [Sch]. The result is as follows:

$$
H_{j}\left(\Lambda\left(S^{n}\right)\right)= \begin{cases}Z ; & j=0,(2 i-1)(n-1),(2 i-1)(n-1)+1 \\ Z_{2} ; & j=2 i(n-1)+1 \\ 0 ; & \text { otherwise }\end{cases}
$$

Since there is constructed Klingenberg homotopy $w_{i}^{\prime}$ for a rational generator $w_{i}$ of $H_{(2 i-1)(n-1)}\left(\Lambda\left(S^{n}\right)\right)$ so that

$$
w_{i}+\vartheta w_{i}=\partial w_{i}^{\prime}
$$

we see that for any cycle $Z$ in $\Lambda\left(S^{n}\right)$ of dimension $(2 i-1)(n-1)$, there exists a chain $Y_{1}$ or $Y_{2}$ in $\Lambda\left(S^{n}\right)$ such that

$$
Z=\partial Y_{1} \text { or } Z+\vartheta Z=\partial Y_{2}
$$

respectively.

In general we can use the fact that $H_{j}\left(\Lambda\left(S^{n}\right)\right)$ is generated by at most a single generator $v_{j}$ for each dimension to find a chain $y_{j}$ which satisfies

$$
v_{\jmath} \pm \vartheta v_{j}=\partial y_{j}
$$

Therefore we have

Proposition 1. For each cycle $Z$ on $\Lambda\left(S^{n}\right)$, we have one of the following possibilities:

1) for a chain $Y_{1}, \quad Z=\partial Y_{1}$

2) for a chain $Y_{2}, \quad Z+\vartheta Z=\partial Y_{2}$

3) for a chain $Y_{3}, \quad Z-\vartheta Z=\partial Y_{3}$,

here we note that we may take $Z$, and $Y_{i}$ as a cycle and chains in the abstract Morse complex, because of Theorem 1.

Identifying summit base to closed geodesic by Proposition 1.2, we start with any closed geodesic $c$. First we see from Theorems 1, 2 that there is a closed geodesic $c_{-}$of 1 (or 2) less index on $\partial c$ such that $m\left(c_{-}\right) \mid m(c)$ or there is a connected cycle $Z(c)$ with non zero barycenter over $c$. Then by Proposition 1 we have a bounding chain $Y_{1}, Y_{2}$ or $Y_{3}$ such that 


$$
Z_{1}=\partial Y_{1}, \quad Z_{2}=Z+\vartheta Z=\partial Y_{2} \quad \text { or } \quad Z_{3}=Z-\vartheta Z=\partial Y_{3} .
$$

By the operator $J_{0}$, we realize $Y_{i}\left(i=1,2\right.$ or 3 ) by a submanifold $J_{0}\left(Y_{i}\right)$ in $A\left(S^{n}\right)$ so that

$$
Z_{i}=K\left(\partial\left(J_{0}\left(Y_{i}\right)\right)\right) \quad(i=1,2 \text { or } 3) .
$$

Since $B_{c}(\vartheta Z(c))=0$, Theorems 1,2 imply the divisibility by $c^{\prime}$ with 1 higher index, provided $Y_{i}$ has no tunnel piece of non zero barycenter.

Hence we have the following first version of the divisibility lemma:

Proposition 2. If it is possible to modify the bounding chain $Y_{i}$ for any cycle $Z_{i}$ so as to $Y_{i}$ does not contain any tunnel piece of non zero barycenter then we see that for any closed geodesic c on $S^{n}$, one of the following two possibility holds:

1) There is a closed geodesic $c_{-}$of 1 or 2 less index so that

$$
m\left(c_{-}\right) \mid 2 m(c), \quad c_{-} \in \partial c .
$$

2) There is a closed geodesic $c^{\prime}$ of 1 higher index so that

$$
m\left(c^{\prime}\right) \mid 2 m(c), \quad c \in \partial c^{\prime} .
$$

The multiple 2 comes from the orientation in the unstable manifold (see Proposition 1, in §2).

The proof of the assumption part in Proposition 2 will be given in Part 2, and also in the appendix we give only a rough sketch under no twisting assumption.

\section{Appendix. A rough sketch of the tunnel killing process}

For the sake of simplicity, we call a submanifold of $\Lambda M$ is transversal if it is transversal to any stable manifold in $\Lambda M$ and we understand submanifold are orientable and may have boundary, unless otherwise specified.

For a transversal submanifold $A$ we define a class $R(A)$ of transversal submanifolds as the totality of compact transversal submanifold $X$ of 1 higher dimension than that of $A$ satisfying $K(\partial X)=K(A)$.

We say for a chain $c$ in Morse complex of $\Lambda M$ that $c$ is realized by a submanifold, if there exists a transversal submanifold $X$ of the same dimensionality as $c$ in $\Lambda M$ such that $K(X)=c$.

Then by taking suitable copies of unstable manifolds to see the following is easy, (see [K-S]). 
Lemma 1. A chain c of Morse complex can be realized by a submanifold.

Corollary 1. For a cycle $Z$ of Morse complex the submanifold realization $X$ of $Z$ can be so chosen that $X$ is connected and $X \in R(\phi)$.

This is obtained by attaching 1 handles killing disconnectivity of $X_{0}$ and the critical points on $\partial X_{0}$ at the same time to the realization $X_{0}$ of Lemma 1 ([K-S]).

Corollary 2. If $\pi_{1}(A M)=0$ then the realization $X$ of a cycle $Z$ in Corollary 1 can be chosen so that $\pi_{1}(X)=0$ for any $Z$ of $\operatorname{dim} Z \geqq 4$.

In fact, obviously attaching 2 handles kills 1 st homotopy of $X_{0}$ of Corollary 1. Since the handles are obtained by the thickening of 2 disks attached to $X_{0}$ at its boundary, we may avoid intersections of the handles with any stable manifold of the critical points of dimension 3 shifting the disks into transversal position.

A submanifold $X$ in $A M$ is said to admit tunnel splitting if there exist disjoint open sets $S(X), T(X)$ in $X$ so that $S^{1}$ action defines a foliated structure on $T(X), S(X)$ is not tangential in $S^{1}$ direction to any (weak) stable manifold $\sigma S(c)$ and the submanifolds $S(X), \bar{T}(X)$ with boundary satisfy that

$$
\overline{S(X)} \cup T(X)=X .
$$

From the construction of realizations, we deduce easily the following:

Lemma 2. All the realizations in Lemma 1, Corollaries 1, 2 of a chain in Morse complex admit the tunnel splitting.

In the rest of this section, we assume that any summit piece $c$ has no tunnel on its boundary which we refer as no twisting property.

LEMma 3. If a submanifold $X$ admits a tunnel splitting and if $\pi_{1}(X)$ $=0$, then the foliated structure on, $T(X)$ is trivial.

In fact, if $\partial T \neq \phi$ then as a closed subset of a compact set, $\partial T$ is compact and split into 2 connected components $B_{+}, B_{-}$, because of orientability of $T$ and of no twisting property.

Since the foliated structure is deduced from $S^{1}$ action, it has the special coordinate coming from $S^{1}$ action and therefore is non compressible. Thus around the closed leaf $B_{+}$or $B_{-}$, it is obviously trivial. Continuation of the process yields Lemma. 
Corollary 3. Under the same assumption as in Lemma 3, we see that any connected component $C$ of the tunnel part of the tunnel splitting is diffeomorphic to $B \times[0, \theta]$ for some $\theta \in S^{1}$, in particular the boundary $\partial C$ of $C$ consists of 2 components $B_{+}, B_{-}$so that

$$
K\left(B_{+}\right)=\theta \circ K\left(B_{-}\right) \text {. }
$$

The following is obvious from the duality, independent of no twisting property;

Lemma 4. Suppose a compact submanifold $X$ contains a closed connected submanifold $B$ of codimension 1 . If $\pi_{1}(X)=0$, then $X-B$ splits into 2 disjoint open sets.

Proposition 1. Assume no twisting property and take a submanifold $X$ bounding connected submanifold $Z$ which has no tunnel in it. If $X$ admits a tunnel splitting and if $\pi_{1}(X)=0$, then we can kill tunnel part from $X$, that is, we can construct a submanifold $X^{1}$ bounding $Z$ so that $K_{T}(X)=0$.

In fact Corollary 3 implies any connected component $C$ is of the form $B \times[0, \theta]$ and has no intersection with $Z$. Thus Lemma 4 yields that $X-C$ splits into 2 disjoint sets $X_{+}, X_{-}$, one of which contain $Z, X_{-} \supset Z$ e.g. Thus take $X_{-} \cup \theta^{-1} \circ X_{+}$and attach handles along the gradient flow to have a manifold $X^{\prime}$ bounding $Z$ again. Since $\pi_{1}\left(X^{\prime}\right)=0$, we may continue this process until we kill all the tunnel part from $X$.

Combining Proposition 1, Lemma 2 and Proposition 3 of Section 2 of Part 1, we finish the proof of Divisibility Lemma under the assumption of no twisting property.

Remark. The tunnel piece on the boundary of a critical point appears in a particular way. If this happens, $S(c)$ looses its transversality to $S^{1}$ action on its boundary, in contrast with the usual tunnel piece. Therefore taking a special consideration on this fact we may kill the tunnels in a similar way as above assuming no twisting property only for 2 dimensional summits, all these will be done in the following part.

\section{REFERENCES}

[H] S. H. Hong, On equivariant Morse complex (to appear).

[K] W. Klingenberg, Lectures on closed geodesics, Springer Verlag 1978. 
[K-S] W. Klingenberg and Y. Shikata, On a proof of divisibility lemma, Moscov Topology Symposium 1980 (also correction in 1984).

[Sch] A. Schwarz, Homology of the space of closed curves, Trudy Moscov. Mat Obšč, 9 (1960), 3-44.

Y. Shikata

Department of Mathematics

Faculty of Sciences

Nagoya University

Chikusa-ku, Nagoya 464

Japan

W. Klingenberg

Mathematisches Institut

der Universität

Wegelerstrasse 10

5300 Bonn

Germany 